\title{
Clinical Study \\ Gender Differences of Plaque Characteristics in Elderly Patients with Stable Angina Pectoris: An Intravascular Ultrasonic Radiofrequency Data Analysis
}

\author{
Tomohiro Nakamura, ${ }^{1}$ Manabu Ogita, ${ }^{1}$ Junya Ako, ${ }^{2}$ and Shin-ichi Momomura ${ }^{2}$ \\ ${ }^{1}$ Cardiovascular Division, Saitama Citizens Medical Center, 299-1 Shimane, Nishi-ku, Saitama 331-0054, Japan \\ ${ }^{2}$ Cardiovascular Division, Saitama Medical Center, Jichi Medical University, Saitama, Japan \\ Correspondence should be addressed to Tomohiro Nakamura, tomonaka@m3.gyao.ne.jp
}

Received 17 May 2010; Accepted 1 December 2010

Academic Editor: Albert G. Hakaim

Copyright (C) 2010 Tomohiro Nakamura et al. This is an open access article distributed under the Creative Commons Attribution License, which permits unrestricted use, distribution, and reproduction in any medium, provided the original work is properly cited.

\begin{abstract}
The purpose of this study was to evaluate the relation between gender and plaque characteristics assessed by virtual histologyintravascular ultrasound (VH-IVUS) in patients with stable angina pectoris. Preinterventional VH-IVUS image was available for analysis in 88 men and 60 women patients. Women had significantly smaller vessel volume $\left(12.7 \pm 3.9\right.$ versus $14.5 \pm 4.2 \mathrm{~mm} / \mathrm{mm}^{3}$, $P=.01)$ and smaller plaque volume $\left(8.4 \pm 3.5\right.$ versus $\left.9.7 \pm 3.5 \mathrm{~mm}^{3} / \mathrm{mm}, P=.04\right)$. However, these differences were no longer significant when corrected for body surface area (BSA). In VH-IVUS analysis, women had significantly higher dense calcium when corrected for BSA in the culprit lesion (volume: $0.32 \pm 0.26$ versus $0.44 \pm 0.40 \mathrm{~mm}^{3} / \mathrm{mm} / \mathrm{BSA}, P=.03$; proportion: $8.2 \pm 6.1$ versus $11.2 \pm 7.6 \%, P=.009)$. VH-IVUS analysis of plaque components in elderly patients with stable angina showed that women had higher calcium contents compared with men.
\end{abstract}

\section{Introduction}

Women differ from men in coronary artery disease epidemiology, symptoms, pathophysiology, and clinical outcome [1-3]. Several studies have reported that women are associated with an increased mortality rate following coronary interventions compared with men. The worse outcomes in women might be partly explained by some clinical factors such as delayed onset of disease, older age, smaller body surface area, smaller vessel, and comorbidities. However, whether women have smaller vessel and different plaque characteristics on atherosclerotic segment have not been clearly documented. And it remains uncertain whether plaque components of coronary stenotic lesion differ between men and women. Recently, virtual histologyintravascular ultrasound (VH-IVUS) using spectral analysis of the radiofrequency ultrasound backscatter signals was introduced to clinical practice to characterize the plaque composition [4]. The purpose of this study was to examine the gender differences of the plaque characteristics in culprit lesions of stable angina patients as assessed by VH-IVUS.

\section{Methods}

Between January 2006 and December 2006, preinterventional VH-IVUS was performed in 240 patients with stable angina undergoing elective coronary intervention. Stable angina was defined as no change in the frequency and duration of cardiac ischemic symptoms within 4 weeks before the intervention. In the present study, inclusion criteria were defined as follows: (1) a de novo lesion with $>75 \%$ angiographic stenosis and (2) over 60-year old patients for matching age difference between gender. We excluded patients with bypass graft lesions, hemodialysis, acute coronary syndrome, or old myocardial infarction. Finally, 148 patients (88 men and 60 women) with stable angina were eligible and enrolled in this study. Written informed consent was obtained from all patients. 
Preinterventional IVUS images were performed after administration of nitroglycerin $200 \mu \mathrm{g}$. A $20 \mathrm{MHz}, 3.2 \mathrm{~F}$, VH-IVUS catheter (Eagle Eye Gold, Volcano Therapeutics, Rancho Cordova, CA, USA) was placed distal to the target lesion and was pulled back with a motorized transducer pullback system at a rate of $0.5 \mathrm{~mm} / \mathrm{s}$. A single observer who was blinded to the clinical/angiographic findings analyzed IVUS images of culprit segment with $>50 \%$ diameter stenosis. Manual contour detection of both the lumen and the mediaadventitia interface was performed. Volumetric data were automatically determined by the software, a summation of measured cross-sectional areas in all frames of the pullback region based on Simpson's rule. The raw radiofrequency data was captured at the top of the $R$ wave during auto-pullback and reconstructed the color-coded map that classified coronary plaque into 4 different components automatically by the software (IVUS Lab Software, Volcano Therapeutics). And the volume and percentage of each plaque component were also automatically calculated by the software. Fibrous tissue was shown in green, fibrofatty tissue in greenishyellow, dense calcium in white, and necrotic core in red based on mathematical autoregressive spectral analysis of IVUS backscatter [4]. The accuracy for each plaque component between in vivo VH-IVUS and in vitro histopathology has been reported to be $87-97 \%$ [5]. Plasma high-sensitive Creactive protein (hs-CRP) was measured by ELISA using an immunonephelometric assay kit (Dade Behring, Illinois). Glomerular filtration rate (GFR) was calculated by Modification of Diet in Renal Disease Study equation [6]. Statistical analysis was performed with StatView (SAS Institute, Cary, North Carolina). Continuous variables were compared by unpaired Student's $t$-test or Mann-Whitney $U$ statistic test. Categorical variables were compared by the chi-square analysis. Volumetric IVUS data was presented as total volume per lesion length $\left(\mathrm{mm}^{3} / \mathrm{mm}\right)$ for correcting the differences of lesion length among the subjects. For all analyses, a $P$ value $<.05$ was defined as statistically significant.

\section{Results}

Women had less diabetes and had higher level of highdensity lipoprotein (Table 1). No women received hormone replacement therapy in the present study. The quantitative analysis of grey scale is shown in Table 2. Women had significantly smaller vessel volume compared with men (women versus men; $12.7 \pm 3.9$ versus $14.5 \pm 4.2 \mathrm{~mm}^{3} / \mathrm{mm}$, $P=.01$ ) and smaller plaque volume (women versus men; $8.4 \pm 3.5$ versus $\left.9.7 \pm 3.5 \mathrm{~mm}^{3} / \mathrm{mm}, P=.04\right)$. However, these differences were no longer significant when corrected for body surface area (BSA). In VH-IVUS analysis after correcting for BSA (Table 3), the volume and percentage of dense calcium were significantly higher in women compared with men (women versus men; $0.44 \pm 0.40$ versus $0.32 \pm$ $0.26 \mathrm{~mm}^{3} / \mathrm{mm} /$ BSA $P=.03,11.2 \pm 7.6$ versus $8.2 \pm 6.1 \%$, $P=.009)$. Though there was no significant difference in the volume of fibrofatty tissue between women and men (women versus men; $0.50 \pm 0.39$ versus $0.62 \pm 0.50 \mathrm{~mm}^{3} / \mathrm{mm} / \mathrm{BSA}$, $P=.11$ ), the percentage of fibrofatty tissue was significantly less in women (women versus men; $12.1 \pm 5.4$ versus 15.1 $\pm 8.7 \%, P=.03$ ). In multivariate analysis (Table 4 ), gender was the only independent factor for the percentage of dense calcium (odds ratio $=2.5, P=.05$ ).

\section{Discussion}

In the present study, using preinterventional VH-IVUS images, women had a higher amount of calcium in the culprit lesion plaque compared with men, though there were no quantitative differences of plaque burden when adjusted for body surface area.

Several studies suggest that women are associated with an increased mortality rate following coronary interventions compared with men, although not consistently observed [7-10]. The worse outcomes in women might be partly explained by some clinical factors such as older age, smaller body surface area, and comorbidities at the time of presentation [11]. However, whether women have different plaque characteristics on atherosclerotic segment has not been clearly documented. An angiographic study in patients without visual evidence of coronary atherosclerosis has reported that lumen size in women was smaller than in men [12], and an autopsy study has reported that this difference in coronary size is due to the mass of the heart [13]. A previous study using grey-scale IVUS has reported that women and men also had similar reference and plaque burden and eccentricity [14]. Similarly, in our study, there were not any gender-specific differences of quantitative variables such as vessel volume and plaque volume when adjusted for body surface area. Taken together with our results, the gender differences of vessel size might be dependent on body size.

Coronary calcified plaques presumably reflect the pathological process of plaque formation and progression from simple fatty streaks to complex plaques [15-17] and are associated with the long-term mortality and ischemic event $[18,19]$. The use of computed tomography for detection of coronary artery calcium has been studied previously, and this method could evaluate the whole coronary arteries and nonculprit segments [20]. Whereas a study using multidetector-row computed tomography has reported that calcium concentration of individual calcified plaque is independent of age and sex [17], our data using VH-IVUS showed that women have more calcified plaque components in the culprit lesion compared with men. It is difficult to distinguish between intimal atherosclerotic calcification and medial calcification by the present imaging modality like CT, grey-scale IVUS, and VH-IVUS. Further studies will be needed to clarify the impact of gender differences on atherosclerotic calcification.

Estrogen has multiple biologic effects that might vary according to the underlying state of the vasculature and other tissue $[21,22]$. A recent randomized clinical study has reported that estrogen could reduce calcified plaque burden of coronary arteries in young menopausal women [23]. Though there was no women who received hormone replacement therapy in the present study, these results might have suggested that estrogen inhibits the progression of atherosclerosis. The biological explanations for gender differences in cardiovascular diseases are more complex, so 
TABLE 1: Baseline clinical characteristics.

\begin{tabular}{|c|c|c|c|}
\hline & $\operatorname{Men}(n=88)$ & Women $(n=60)$ & $P$ value \\
\hline Age, $y$ & $71 \pm 6$ & $70 \pm 6$ & .47 \\
\hline Diabetes, $n(\%)$ & $33(38)$ & $13(22)$ & .05 \\
\hline Hyperlipidemia, $n(\%)$ & $47(53)$ & $45(75)$ & .18 \\
\hline Hypertension, $n(\%)$ & $67(63)$ & $37(62)$ & .08 \\
\hline Current smoking, $n(\%)$ & $41(47)$ & $8(13)$ & .0001 \\
\hline Family history of CAD, $n(\%)$ & $17(19)$ & $13(22)$ & .69 \\
\hline $\mathrm{LDL}, \mathrm{mg} / \mathrm{dL}$ & $112.9 \pm 28.0$ & $122.0 \pm 30.7$ & .06 \\
\hline $\mathrm{HDL}, \mathrm{mg} / \mathrm{dL}$ & $45.1 \pm 9.4$ & $51.6 \pm 12.2$ & .0004 \\
\hline HbAlc, \% & $6.1 \pm 1.3$ & $5.7 \pm 1.1$ & .06 \\
\hline Statin use, $n(\%)$ & $29(33)$ & $16(27)$ & .52 \\
\hline Insulin use, $n(\%)$ & $10(11)$ & $5(8)$ & .75 \\
\hline $\mathrm{GFR}, \mathrm{mL} / \mathrm{min}$ & $65.7 \pm 20.0$ & $67.3 \pm 17.2$ & .62 \\
\hline hs-CRP, mg/L & $1.7 \pm 1.6$ & $2.1 \pm 2.9$ & .89 \\
\hline Vessel & & & .15 \\
\hline Left anterior descending & $41(47)$ & $30(50)$ & \\
\hline Left circumflex & $17(19)$ & $17(28)$ & \\
\hline Right coronary artery & $30(34)$ & $12(20)$ & \\
\hline Triple vessel disease, $n(\%)$ & $25(28)$ & $17(28)$ & $>.99$ \\
\hline Lesion length, mm & $22.0 \pm 10.8$ & $18.3 \pm 10.8$ & .07 \\
\hline
\end{tabular}

Data are presented as mean \pm SD or number of patients (\%). CAD: coronary artery disease; LDL: low-density lipoprotein; HDL: high-density lipoprotein; GFR: glomerular filtration rate; hs-CRP: high-sensitive C-reactive protein.

TABLE 2: Grey-scale IVUS analysis.

\begin{tabular}{lccc}
\hline & Men $(n=88)$ & Women $(n=60)$ & $P$ value \\
\hline Vessel volume, $\mathrm{mm}^{3} / \mathrm{mm}$ & $14.5 \pm 4.2$ & $12.7 \pm 3.9$ & .01 \\
Lumen volume, $\mathrm{mm}^{3} / \mathrm{mm}$ & $4.8 \pm 1.0$ & $4.5 \pm 1.3$ & .07 \\
Plaque volume, $\mathrm{mm}^{3} / \mathrm{mm}$ & $9.7 \pm 3.5$ & $8.4 \pm 3.5$ & .04 \\
Vessel volume, $\mathrm{mm}^{3} / \mathrm{mm} / \mathrm{BSA}$ & $8.8 \pm 2.6$ & $8.5 \pm 2.6$ & .64 \\
Lumen volume, $\mathrm{mm}^{3} / \mathrm{mm} / \mathrm{BSA}$ & $2.9 \pm 0.7$ & $2.9 \pm 0.7$ & .83 \\
Plaque volume, $\mathrm{mm}^{3} / \mathrm{mm} / \mathrm{BSA}$ & $5.8 \pm 2.2$ & $5.8 \pm 2.2$ & .81 \\
\hline
\end{tabular}

Data are presented as mean \pm SD. EEM: external elastic membrane, BSA: body surface area.

TABLE 3: Virtual histology-IVUS analysis.

\begin{tabular}{|c|c|c|c|}
\hline & $\operatorname{Men}(n=88)$ & Women $(n=60)$ & $P$ value \\
\hline Fibrous plaque volume, $\mathrm{mm}^{3} / \mathrm{mm} / \mathrm{BSA}$ & $2.66 \pm 1.58$ & $2.33 \pm 1.41$ & .20 \\
\hline Fibrofatty plaque volume, $\mathrm{mm}^{3} / \mathrm{mm} / \mathrm{BSA}$ & $0.62 \pm 0.50$ & $0.50 \pm 0.39$ & .11 \\
\hline Necrotic core plaque volume, $\mathrm{mm}^{3} / \mathrm{mm} / \mathrm{BSA}$ & $0.53 \pm 0.31$ & $0.59 \pm 0.37$ & .26 \\
\hline Dense calcium plaque volume, $\mathrm{mm}^{3} / \mathrm{mm} / \mathrm{BSA}$ & $0.32 \pm 0.26$ & $0.44 \pm 0.40$ & .03 \\
\hline$\%$ Fibrous plaque, $\%$ & $63.1 \pm 9.8$ & $61.3 \pm 12.5$ & .09 \\
\hline$\%$ Fibrofatty plaque, $\%$ & $15.1 \pm 8.7$ & $12.1 \pm 5.4$ & .03 \\
\hline$\%$ Necrotic core, $\%$ & $13.6 \pm 7.2$ & $15.4 \pm 6.0$ & .12 \\
\hline$\%$ Dense calcium, \% & $8.2 \pm 6.1$ & $11.2 \pm 7.6$ & .009 \\
\hline
\end{tabular}

Data are presented as mean \pm SD. BSA: body surface area.

we think that the synthesis of the underlying mechanisms that explain these differences has been impossible.

Several limitations should be noted. This is a study with a limited number of patients with stable angina undergoing coronary intervention, possibly posing a risk of patient selection bias. Our analysis included only the culprit lesions; therefore, the results may not be applicable to other parts of coronary arteries. The VH-IVUS technology is unable to differentiate thrombus from other plaque are components. Although we have investigated the patients with stable 
TABLE 4: Multivariate analysis.

\begin{tabular}{llcc}
\hline & & OR $(95 \%$ CI $)$ & $P$ value \\
\hline $\begin{array}{l}\text { \% Dense } \\
\text { calcium versus }\end{array}$ & Gender (women) & $2.5(0.4-4.9)$ & .05 \\
& Diabetes & $1.4(-1.1-3.9)$ & .28 \\
& Hyperlipidemia & $0.6(-1.7-2.9)$ & .60 \\
& Hypertension & $0.6(-1.9-3.1)$ & .64 \\
& Current smoking & $-1.8(-4.4-0.8)$ & .18 \\
\hline
\end{tabular}

OR: odds ratio, CI: confidence interval.

angina, there is a possibility that small thrombi in the plaque classified into the incorrect tissue. The differences in comorbid conditions and lipid profiles may have influenced the results. In previous studies using VH-IVUS analysis, the presence of diabetes and lower HDL level contributes to the increment of dense calcium and necrotic core [24, 25]. Although diabetes patients were less and high-density lipoprotein levels were higher in women, absolute and percentage of dense calcium volume were higher in women compared with men. And gender was an independent factor for the percentage of dense calcium in multivariate analysis. Thus, we think that gender difference might contribute to the calcification of coronary arteries independently. As we have also excluded the patients younger than 60 years old, these results cannot be generalized to all age groups of women due to the different risk profile in pre- versus postmenopausal women.

In conclusion, the present study using preinterventional VH-IVUS images has suggested that women had more calcium in the composition of the culprit lesion plaque compared with men in elderly patients with stable angina. While it is yet to be proven if the management of coronary calcification has a significant clinical relevance, further studies with a gender-specific approach are necessary for the better understanding of coronary heart disease.

\section{References}

[1] C. J. Pepine, "Ischemic heart disease in women: facts and wishful thinking," Journal of the American College of Cardiology, vol. 43, no. 10, pp. 1727-1730, 2004.

[2] C. J. Pepine, R. S. Balaban, R. O. Bonow et al., "Women's Ischemic Syndrome Evaluation: current status and future research directions: report of the National Heart, Lung and Blood Institute workshop: October 2-4, 2002: section 1: diagnosis of stable ischemia and ischemic heart disease," Circulation, vol. 109, no. 6, pp. e44-e46, 2004.

[3] A. Bellasi, P. Raggi, C. N. B. Merz, and L. J. Shaw, "New insights into ischemic heart disease in women," Cleveland Clinic Journal of Medicine, vol. 74, no. 8, pp. 585-594, 2007.

[4] A. Nair, B. D. Kuban, E. M. Tuzcu, P. Schoenhagen, S. E. Nissen, and D. G. Vince, "Coronary plaque classification with intravascular ultrasound radiofrequency data analysis," Circulation, vol. 106, no. 17, pp. 2200-2206, 2002.

[5] K. Nasu, E. Tsuchikane, O. Katoh et al., "Accuracy of in vivo coronary plaque morphology assessment: a validation study of in vivo virtual histology compared with in vitro histopathology," Journal of the American College of Cardiology, vol. 47, no. 12, pp. 2405-2412, 2006.

[6] A. S. Levey, J. P. Bosch, J. B. Lewis, T. Greene, N. Rogers, and D. Roth, "A more accurate method to estimate glomerular filtration rate from serum creatinine: a new prediction equation. Modification of Diet in Renal Disease Study Group," Annals of Internal Medicine, vol. 130, pp. 461-470, 1999.

[7] F. Alfonso, R. Hernández, C. Bañuelos et al., "Initial results and long-term clinical and angiographic outcome of coronary stenting in women," American Journal of Cardiology, vol. 86, no. 12, pp. 1380-1383, 2000.

[8] J. Mehilli, A. Kastrati, J. Dirschinger, H. Bollwein, F. J. Neumann, and A. Schömig, "Differences in prognostic factors and outcomes between women and men undergoing coronary artery stenting," Journal of the American Medical Association, vol. 284, no. 14, pp. 1799-1805, 2000.

[9] C. T. Watanabe, C. Maynard, and J. L. Ritchie, "Comparison of short-term outcomes following coronary artery stenting in men versus women," American Journal of Cardiology, vol. 88, no. 8, pp. 848-852, 2001.

[10] A. J. Lansky, J. S. Hochman, P. A. Ward et al., "Percutaneous coronary intervention and adjunctive pharmacotherapy in women: a statement for healthcare professionals from the American Heart Association," Circulation, vol. 111, no. 7, pp. 940-953, 2005.

[11] A. K. Jacobs, "Coronary revascularization in women in 2003: sex revisited," Circulation, vol. 107, no. 3, pp. 375-377, 2003.

[12] J. T. Dodge, B. G. Brown, E. L. Bolson, and H. T. Dodge, "Lumen diameter of normal human coronary arteries: influence of age, sex, anatomic variation, and left ventricular hypertrophy or dilation," Circulation, vol. 86, no. 1, pp. 232246, 1992.

[13] C. S. Roberts and W. C. Roberts, "Cross-sectional area of the proximal portions of the three major epicardial coronary arteries in 98 necropsy patients with different coronary events. Relationship to heart weight, age and sex," Circulation, vol. 62, no. 5, pp. 953-959, 1980.

[14] R. Kornowski, A. J. Lansky, G. S. Mintz et al., "Comparison of men versus women in cross-sectional area luminal narrowing, quantity of plaque, presence of calcium in plaque, and lumen location in coronary arteries by intravascular ultrasound in patients with stable angina pectoris," American Journal of Cardiology, vol. 79, no. 12, pp. 1601-1605, 1997.

[15] G. C. Mautner, S. L. Mautner, J. Froehlich et al., "Coronary artery calcification: assessment with electron beam CT and histomorphometric correlation," Radiology, vol. 192, no. 3, pp. 619-623, 1994.

[16] G. Sangiorgi, J. A. Rumberger, A. Severson et al., "Arterial calcification and not lumen stenosis is highly correlated with atherosclerotic plaque burden in humans: a histologic study of 723 coronary artery segments using nondecalcifying methodology," Journal of the American College of Cardiology, vol. 31, no. 1, pp. 126-133, 1998.

[17] F. Moselewski, C. J. O’Donnell, S. Achenbach et al., "Calcium concentration of individual coronary calcified plaques as measured by multidetector row computed tomography," Circulation, vol. 111, no. 24, pp. 3236-3241, 2005.

[18] M. J. Budoff, L. J. Shaw, S. T. Liu et al., "Long-term prognosis associated with coronary calcification: observations from a registry of 25,253 patients," Journal of the American College of Cardiology, vol. 49, no. 18, pp. 1860-1870, 2007. 
[19] A. B. Newman, B. L. Naydeck, D. G. Ives et al., "Coronary artery calcium, carotid artery wall thickness, and cardiovascular disease outcomes in adults 70 to 99 years old," American Journal of Cardiology, vol. 101, no. 2, pp. 186-192, 2008.

[20] M. Hosoi, T. Sato, K. Yamagami et al., "Impact of diabetes on coronary stenosis and coronary artery calcification detected by electron-beam computed tomography in symptomatic patients," Diabetes Care, vol. 25, no. 4, pp. 696-701, 2002.

[21] J. E. Manson, S. S. Bassuk, S. M. Harman et al., "Postmenopausal hormone therapy: new questions and the case for new clinical trials," Menopause, vol. 13, no. 1, pp. 139-147, 2006.

[22] M. E. Mendelsohn and R. H. Karas, "Molecular and cellular basis of cardiovascular gender differences," Science, vol. 308, no. 5728, pp. 1583-1587, 2005.

[23] J. E. Manson, M. A. Allison, J. E. Rossouw et al., "Estrogen therapy and coronary-artery calcification," New England Journal of Medicine, vol. 356, no. 25, pp. 2591-2602, 2007.

[24] E. Missel, G. S. Mintz, S. G. Carlier et al., "In vivo virtual histology intravascular ultrasound correlates of risk factors for sudden coronary death in men: results from the prospective, multi-centre virtual histology intravascular ultrasound registry," European Heart Journal, vol. 29, no. 17, pp. 2141-2147, 2008.

[25] K. Nasu, E. Tsuchikane, O. Katoh et al., "Plaque characterisation by Virtual Histology intravascular ultrasound analysis in patients with type 2 diabetes," Heart, vol. 94, no. 4, pp. 429433, 2008. 


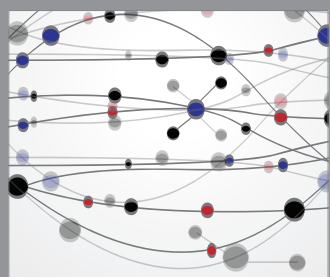

The Scientific World Journal
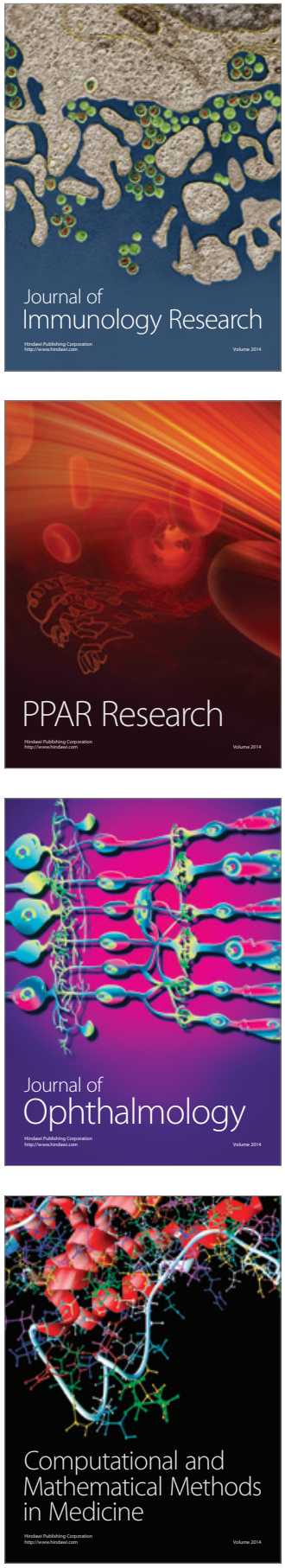

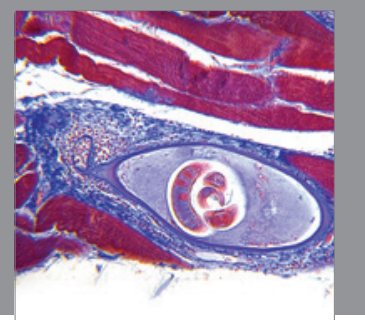

Gastroenterology

Research and Practice
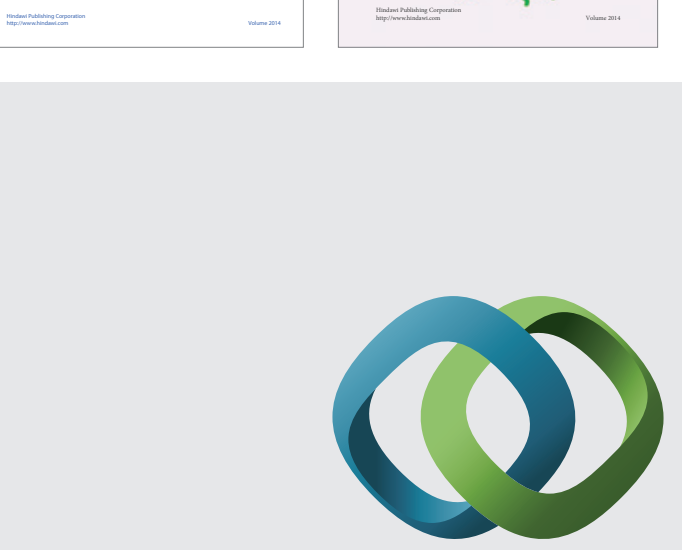

\section{Hindawi}

Submit your manuscripts at

http://www.hindawi.com
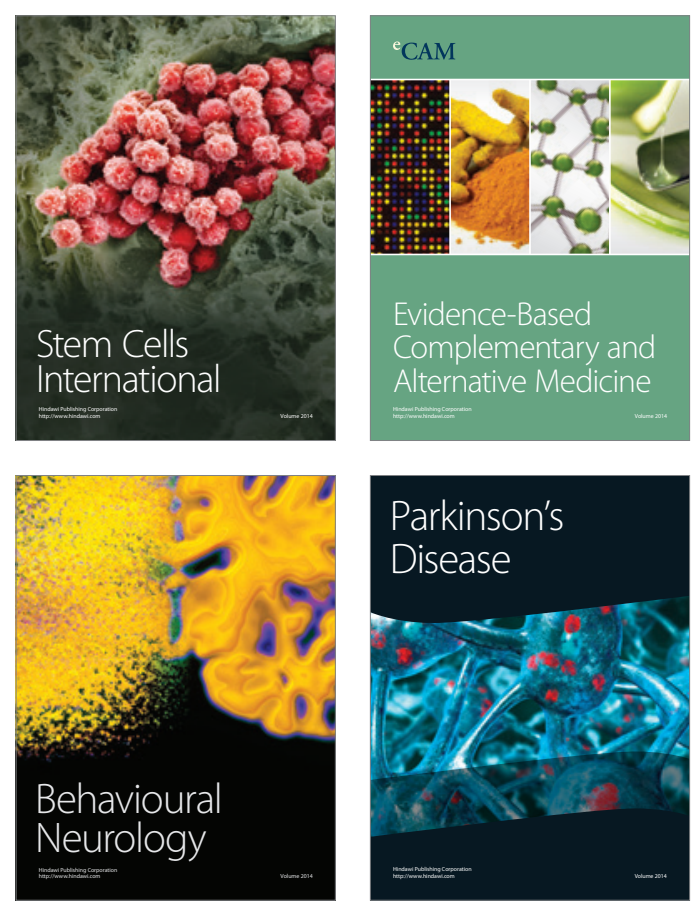

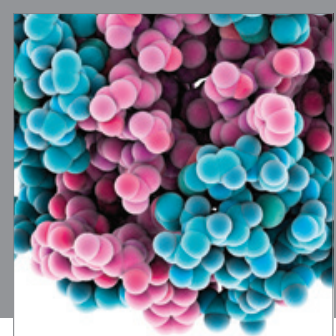

Journal of
Diabetes Research

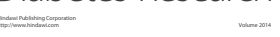

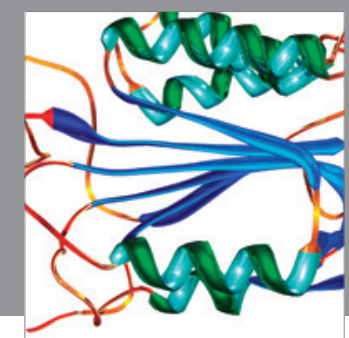

Disease Markers
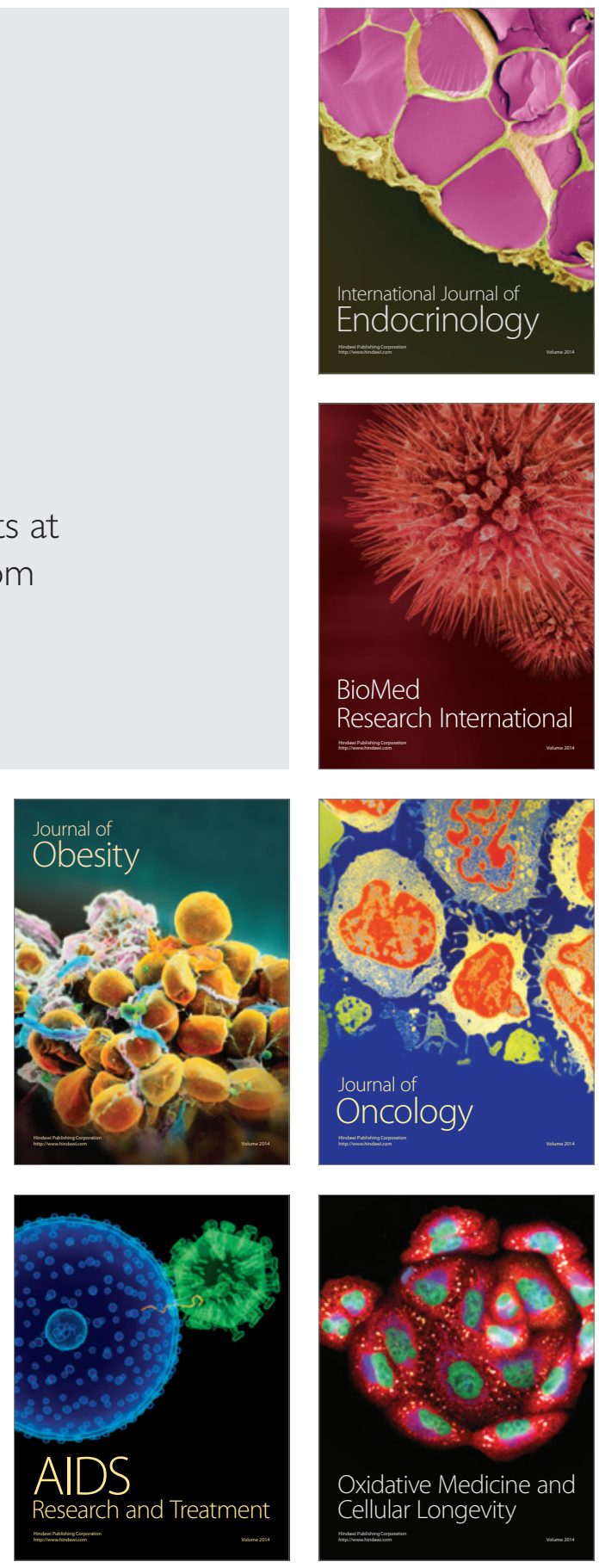